\title{
Analysis of Intensive Learning and Supervised Learning
}

\author{
Qiu Jing \\ JiNan University-University of Birmingham joint institute, Shandong Jinan 511443, \\ China
}

\begin{abstract}
Reinforcement learning is an important branch of machine learning. It is a product of multidisciplinary and multi-domain intersection. Its essence is to solve the decision making problem, that is, to make decisions automatically and to make continuous decisions. It consists of four elements, agent, environmental status, actions, and rewards. The goal of intensive learning is to get the most cumulative rewards. This paper analyzes the definition of reinforcement learning through image, and expounds the difference between reinforcement learning and supervised learning. Finally, several practical applications of reinforcement learning are listed.
\end{abstract}

KEYWORDS: Reinforcement learning, Supervision learning, Application

\section{Introduction}

Reinforcement learning is a reward-guided behavior in which the agent learns in a "trial and error" manner, and the goal is to maximize the reward of the agent through the interaction with the environment. The reinforcement learning is different from the supervised learning in connectedism learning. It is mainly reflected in the teacher's signal. The enhanced signal provided by the environment in reinforcement learning is to evaluate the quality of the action (usually a scalar signal), rather than telling the reinforcement learning system RLS (reinforcement learning system) how to generate the correct action. Because the external environment provides very little information, RLS must learn on its own experience. In this way, RLS gains knowledge in an action-evaluation environment and improves the action plan to adapt to the environment.

\section{Reinforced learning definition}

Let's take a child to learn to walk as an example of an image: a child wants to walk, but before that, he needs to stand up first, and then maintain balance after standing up. Then he has to take a leg first, the left leg. Still right leg, take a step after taking a step. As shown in the following figure, a child is an agent who tries to 
manipulate the environment (walking surface) by taking action (ie walking) and transitions from one state to another (ie, every step he takes) when he completes the task. When a subtask (that is, a few steps), the child gets a reward (to eat chocolate), and when he can't walk, he doesn't give chocolate.

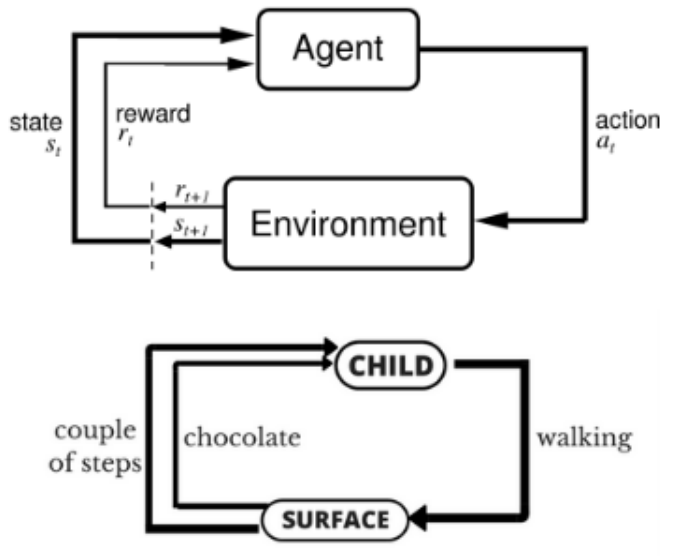

Figure. 1 Example diagram

\section{The difference between reinforcement learning and supervised learning}

Supervised learning is like when you are studying, there is a mentor pointing around, he knows how it is right, but in many practical problems, such as chess, go, there are thousands of combinations. In the case of the way, it is impossible for a tutor to know all possible outcomes.

At this time, reinforcement learning will get a result by first trying to make some behaviors without any label. If the result is right or wrong feedback, adjust the previous behavior, and then adjust continuously, the algorithm can learn the best results can be obtained by what kind of behavior is chosen under what circumstances.

Both learning methods learn a mapping from input to output. Supervised learning is the relationship between the two. It can tell the algorithm what kind of input corresponds to what kind of output, and the reinforcement learning is the feedback function to the machine. Is used to judge whether this behavior is good or bad.

In addition, there is a delay in the feedback of the intensive learning. Sometimes it may take a lot of steps to know whether the choice of a previous step is good or bad, and the poorly chosen choice of supervised learning will be immediately fed back to the algorithm. 
Moreover, the input faced by reinforcement learning is always changing. Whenever an algorithm makes a behavior, it affects the input of the next decision, and the input of supervised learning is independent and identical.

Through reinforcement learning, an agent can make trade-offs between exploration and exploitation and choose the one with the greatest return. Exploration will try a lot of different things to see if they are better than they have tried before. Exploitation will try the most effective behavior in past experience. The general supervised learning algorithm does not consider this balance, it is just an exploitative.

\section{Application examples}

There are many applications for reinforcement learning. In addition to driverless, AlphaGo, and games, there are practical examples of these projects:

\subsection{Manufacturing}

For example, in a Japanese company, Fanuc, when a factory robot picks up an object, it captures the video of the process, remembers its actions every time, the operation succeeds or fails, accumulates experience, and the next time you can take action faster and more accurately. .

\subsection{Inventory Management}

In inventory management, management is a difficult problem because of large inventory, fluctuations in inventory demand, and slow inventory replenishment. It is possible to reduce inventory turnaround time and increase space utilization by establishing a reinforcement learning algorithm.

\subsection{Dynamic pricing}

Q-learning in reinforcement learning can be used to deal with dynamic pricing issues.

\subsection{Customer Delivery}

When transporting to individual customers, the manufacturer wants to reduce the total cost of the fleet while meeting all of the customer's needs. With the multiagents system and Q-learning, you can reduce time and reduce the number of vehicles. 
ISSN 2616-5775 Vol. 1, Issue 1: 80-84, DOI: 10.25236/AJCIS.010008

\subsection{ECommerce Personalization}

In e-commerce, reinforcement learning algorithms can also be used to learn and analyze customer behavior, and to customize products and services to meet the individual needs of customers.

\subsection{Ad Serving}

For example, the algorithm LinUCB (an algorithm that is part of the intensive learning algorithm bandit) will try to deliver a wider range of ads, even though it has not been viewed a lot in the past to better estimate the true click-through rate.

In the case of the double 11 recommendation scenario, Alibaba uses deep reinforcement learning and adaptive online learning to build a decision engine through continuous machine learning and model optimization, real-time analysis of massive user behavior and tens of billions of commodity features to help each user. Quickly discover the baby and improve the efficiency of pairing people and goods. Also, the use of reinforcement learning has increased the click-through rate of mobile phone users by $10-20 \%$.

\subsection{Financial Investment Decisions}

For example, the company Pit.ai, applying reinforcement learning to evaluate trading strategies, can help users build trading strategies and help them achieve their investment goals.

\subsection{Medical Industry}

The Dynamic Therapy Program (DTR) is a topic of medical research aimed at finding effective treatments for patients. For example, cancer, a treatment that requires long-term application, the intensive learning algorithm can use the patient's various clinical indicators as input to develop a treatment strategy.

\section{Conclusion}

Reinforcement learning is a product of multidisciplinary and multi-domain intersections. Its essence is to solve the decision making problem, that is, to make decisions automatically and to make continuous decisions. This paper analyzes the definition of reinforcement learning through image, and expounds the difference between reinforcement learning and supervised learning. Finally, several practical applications of reinforcement learning are listed. 


\section{References}

[1] Lai Wei.Artificial Intelligence: Promoting Urban Transformation and Upgrading [J]. China Automatic Identification Technology. 2018 (02).

[2] Xu Lin.Solving Nonlinear Equations Based on Machine Learning Thoughts [J]. Journal of Heilongjiang Institute of Technology. 2016 (06). 